\title{
Likely Uptake of a Future a Lung Cancer Screening Programme in Hodgkin Lymphoma Survivors: a Questionnaire Study
}

Rachel Broadbent ( $\nabla$ rachel.broadbent-2@postgrad.manchester.ac.uk)

University of Manchester

Christopher J. Armitage

University of Manchester

Philip Crosbie

University of Manchester

John Radford

University of Manchester

Kim Linton

University of Manchester

Research Article

Keywords: Hodgkin lymphoma, Lung cancer, Screening, Willingness

Posted Date: December 13th, 2021

DOI: https://doi.org/10.21203/rs.3.rs-1137564/v1

License: (c) (1) This work is licensed under a Creative Commons Attribution 4.0 International License. Read Full License 


\section{Abstract \\ Background}

Many Hodgkin lymphoma (HL) survivors are at increased risk of subsequent malignant neoplasms (SMN), including lung cancer, due to previous treatment for HL. Lung cancer screening (LCS) detects early-stage lung cancers in ever smokers but HL survivors without a heavy smoking history are ineligible for screening. There is a rationale to develop a targeted LCS. The aim of this study was to investigate levels of willingness to undergo LCS in HL survivors, and to identify the psycho-social factors associated with screening hesitancy.

\section{Methods}

A postal questionnaire was sent to $281 \mathrm{HL}$ survivors registered in a long-term follow-up database and at increased risk of SMNs. Demographic, lung cancer risk factors, psycho-social and LCS belief variables were measured. Multivariable logistic regression analysis was performed to determine the factors associated with lung cancer screening hesitancy, defined as those who would 'probably' or 'probably not' participate.

\section{Results}

The response rate to the questionnaire was $58 \%(n=165)$. Participants were more likely to be female, older and living in a less deprived area than non-participants. Uptake (at any time) of breast and bowel cancer screening among those previously invited was $99 \%$ and $77 \%$ respectively. 159 participants were at excess risk of lung cancer. The following results refer to these 159. Around half perceived themselves to be at greater risk of lung cancer than their peers. Only $6 \%$ were eligible for lung cancer screening pilots aimed at ever smokers in the UK. $98 \%$ indicated they would probably or definitely participate in LCS were it available. Psycho-social variables associated with LCS hesitancy on multivariable analysis were male gender (OR $5.94 \mathrm{Cl} 1.64-21.44, \mathrm{p}<0.01$ ), living in an area with a high index of multiple deprivation (IMD) decile (deciles 6-10) (OR 8.22 Cl 1.59-42.58, p<0.05) and lower levels of self-efficacy (OR 1.64 Cl 1.30-2.08 $\mathrm{p}<0.01)$.

\section{Conclusion}

HL survivors responding to this survey were willing to participate in a future LCS programme but there was some hesitancy. A future LCS trial for HL survivors should consider the factors associated with screening hesitancy in in order to minimise barriers to participation.

\section{Introduction}


Hodgkin lymphoma $(\mathrm{HL})$ is a lymphoid malignancy of clonal B cells predominantly affecting the young and the elderly and accounts for $68 \%$ of lymphomas in $15-24$ year olds. [1], [2] Whilst over $90 \%$ of patients diagnosed under the age of 50 are cured with chemotherapy and/or radiotherapy, five year survival rates fall with increasing age at diagnosis.[1] As a consequence of treatment with alkylating agents and radiation, survivors of $\mathrm{HL}$ are at excess risk of developing subsequent malignant neoplasms (SMN), which are the primary cause of death among long-term survivors. [3][4] The most common SMNs in HL survivors are breast cancer (cumulative incidence $(\mathrm{Cl}) 35$-years post-treatment $14.4 \%$ ) and lung cancer $(\mathrm{Cl}$ 35-years post-treatment 3.1\% in women and 5.2\% in men) [5]. However, the SMNs most commonly associated with mortality are gastrointestinal cancers and lung cancer, with an absolute risk of death of 10.4 and 9.4 respectively.[6], [7] In the case of lung cancer, a large case-control study found the relative risks for lung cancer in HL survivors following alkylating agents, radiation to lung $\geq 5 \mathrm{~Gy}$, or both to be 7.2 , 4.3 and 7.2 respectively in light or never smokers, increasing in a multiplicative fashion to $20.2,16.3$ and 49.1 respectively in moderate to heavy smokers.[3] Despite these excess risks, screening for early detection of SMNs is only available for women at very high risk of breast cancer.[8]

Screening for lung cancer using a low-dose CT scan detects early stage, asymptomatic lung cancers and has been shown to reduce lung cancer mortality in current and former smokers in two large randomised controlled trials.[9], [10] In the United Kingdom (UK), lung cancer screening is being piloted by the National Health Service (NHS) in former or current smokers aged 55-74, whose eligibility for screening is determined using a lung cancer risk calculator.[11] Rates of smoking among HL survivors are low,[12] and since the lung cancer risk calculators aimed at ever smokers do not take into account the risks associated with prior cancer treatment with radiation and alkylating agents, many $\mathrm{HL}$ survivors will not be captured by the lung cancer screening pilots aimed at ever smokers. For this reason, a future lung cancer screening programme for $\mathrm{HL}$ survivors must target this population, much like the approach to breast cancer screening.

The positive attitudes of the general public to cancer screening in the UK [13] are reflected in the relatively high levels of uptake for NHS breast, cervical and bowel cancer screening programmes compared to other countries in Europe. [14] The UK-based Lung Screen Uptake Study reported a 53\% uptake rate, the highest reported rate among historically low uptake rates for lung cancer screening pilots and trials. [15] Uptake of cancer screening is higher among cancer survivors than non-cancer survivors [16], [17]. In a qualitative study, HL survivors were motivated to participate in a future lung cancer screening programme and reported few barriers to participation [18]. However, it is likely that some of the sociodemographic and psychological barriers to cancer screening in the general public will also apply to $\mathrm{HL}$ survivors. This area is worthy of further investigation because uptake of a future targeted lung cancer screening programme could be optimised by interventions designed to minimise known barriers to uptake. In the general population, sociodemographic variables associated with reduced screening participation include older age, male gender and lower socioeconomic status, although the association varies across different screening programmes. Lower levels of education and health literacy - which correlate with lower socioeconomic status - have also been associated with reduced screening participation. [19] 
Theories such as the Health Belief Model (HBM) have been used to explain variation in screening participation. The HBM constructs of perceived susceptibility, perceived severity, perceived benefits and barriers and self-efficacy have been shown to predict cancer screening uptake.[20], [21] Other factors predictive of non-participation in cancer screening programmes include worse self-rated health [22] and lower levels of dispositional optimism, [23] whilst higher levels of cancer worry are both a facilitator and a barrier to participation.[24], [25] Smoking is widely understood by the public as being an important risk factor for lung cancer but current smokers are less likely to participate in lung cancer screening than former smokers.[26], [27] The aim of this study was to use quantitative methods to describe the psychosocial factors associated with hesitancy to participate in a future lung cancer screening programme in $\mathrm{HL}$ survivors.

\section{Methods}

\section{Subjects and setting}

Potential participants were identified from a prospective database of $\geq 5$ year lymphoma survivors (ADAPT) held at The Christie NHS Foundation Trust. HL survivors were eligible for the study if they were currently aged 18-80 and had been treated with chemotherapy regimens that contained alkylating agent/s associated with excess risk of SMN, and/or radiotherapy where the breast/s, lung/s or bowel were included in the radiation field. We excluded those with a diagnosis of dementia, learning difficulties or a SMN for which they were undergoing palliative treatment. A postal questionnaire and participant information sheet was sent to 281 eligible individuals followed by reminder letters to those who had not returned the questionnaire within three weeks. Return of the questionnaire was taken as consent to participate.

\section{Study questionnaire development}

The study questionnaire was designed for use in this study, but used or adapted a number of published scales, as described below. The questionnaire can be found in Supplementary data.

\section{Measures}

\section{Willingness to undergo lung cancer screening}

Participants were asked to rate the strength of their willingness to participate in a future lung cancer screening programme with the question 'If you were invited to go for a lung cancer screening test, would you go?' The response options were 'yes definitely', 'yes probably, 'probably not' and 'definitely not'.

\section{Lung cancer screening related health beliefs}

Lung cancer screening related health beliefs were measured using the Lung Cancer Screening Health Belief Scales (LCSHBS), developed to measure health beliefs impacting lung cancer screening uptake using the HBM framework and psychometrically tested in ever smokers. [28] The LCSHBS comprise of 
four scales measuring perceived risk of developing lung cancer and perceived benefits, perceived barriers and self-efficacy (an individuals' belief in their capacity to execute a behaviour) for undergoing lung cancer screening. Although the Extended Health Belief Model includes separate constructs for perceived risk and perceived severity, the LCSHBS do not include a perceived severity scale because cancer is always perceived to be severe. To adapt the LCSHBS for this study population, items relating to cost, lack of a regular healthcare provider and booking a scan appointment were removed and never smokers were instructed not to complete the items in the perceived barriers scale which relate to a personal history of smoking. Prior to completing the scales, participants were provided with a short statement describing a lung cancer screening test.

Items in the perceived risk, perceived benefits and perceived barriers scales were scored using 5-point Likert scales indicating agreement (strongly agree/agree/neither agree nor disagree/disagree/strongly disagree). The self-efficacy scale had a 4-point Likert scale indicating level of confidence (very confident/somewhat confident/slightly confident/ not at all confident). The following are examples of items included in the scales: 'It is likely that I will get lung cancer in the next five years' (perceived risk scale); 'Having a lung scan would lower my chances of dying from lung cancer', 'Having a lung scan would help me plan for the future' (perceived benefits scale); 'I might put off a lung scan because no one in my family had lung cancer', 'I might put off having a lung scan because I think I am too old to benefit from screening for lung cancer' (perceived barriers scale; 'How confident are you that you could find transportation to get to and from the clinic/hospital to have a lung scan?', 'How confident are you that you could get a lung scan even if you were anxious about the results?' (self-efficacy scale).

Cronbach's alpha was used to estimate internal consistency for each of the LCSHBS subscales and was found to be .90 for the 3 -item perceived risk scale, .84 for 6 -item perceived benefits scale, .89 for the 7 item self-efficacy scale, .94 for the 15-item perceived barrier scale for ever smokers and .91 for the 12item perceived barrier scale for never smokers.

\section{Demographic factors}

Participants' age, gender and full postcode were extracted from electronic medical records. The questionnaire included questions about ethnicity, current employment status and level of education.

\section{Other psychosocial and health related factors}

Cancer worry was measured using an item adapted from the Cancer Worry Chart [29] which is considered to measure cancer worry severity: 'In the last 4 weeks, how often were you bothered by thoughts or worry about your chances of getting cancer again in the future?' (response options not at all / slightly / moderately / quite a bit / extremely).[30] Dispositional optimism was measured using the Revised Life Orientation Test (LOT-R), [31] in which a higher score represents a higher level of dispositional optimism. Self-rated health was measured with a single item taken from the SF-12 Health Survey. [32] Optimistic bias was measured using an existing question relating to developing melanoma [33] adapted for this study: Compared to the average person of your age and sex, how likely is it in your opinion that you will 
develop [lung] cancer? (response options: much less likely / a bit less likely / about the same / a bit more likely / much more likely / I don't know). We developed items to measure presence of a close family history of lung cancer (in parents or siblings), prior uptake of breast or bowel cancer screening and prior knowledge of lung cancer as a late effect of HL treatment. To investigate 6-year lung cancer risk values in our participants, demographic and lung cancer risk factor data were entered into the PLCOall2014 (Prostate, Lung, Colorectal, Ovarian) lung cancer risk calculator. The PLCOall2014 calculator is analogous to the PLCOm2012 calculator designed for ever smokers which is currently used to determine eligibility to undergo lung cancer screening in the UK, however PLCOall2014 also calculates 6-year lung cancer risk in never smokers. When it was used to calculate 6-year lung cancer risk in 65,711 never smokers in the PLCO cohort, the maximum risk observed was $1.47 \%$ falling below the $\geq 1.51 \%$ risk threshold for screening. [34]

\section{Statistical analysis}

Descriptive statistics were used to analyse the demographic and clinical characteristics of participants at risk of lung cancer, their knowledge of lung cancer risk, cancer screening behaviours and future lung cancer screening willingness and responses to the LCSHBS.

The demographic characteristics of participants versus non-participants were compared using Chisquared test for gender and Mann-Whitney U-test for age and Index of Multiple Deprivation (IMD) decile. To identify the psycho-social factors associated with lung cancer screening hesitancy - defined as those responding 'yes probably' or 'probably not' to the lung cancer screening willingness question - a binary logistic regression analysis was performed. The dependent variable was screening willingness and participants for whom complete data was available for the independent variables were included in the analysis. Independent variables included socio-demographics, psychological variables (cancer worry and LOT-R scale score) and LCSHBS scores. Independent variables were entered into the multivariable logistic regression model regardless of whether they were associated with screening hesitancy on univariate analysis.

For the logistic regression, LCSHBS scoring for perceived risk, perceived benefits and perceived selfefficacy was reversed so that higher scores represented lower risk perception, lower perceived benefits and lower-self-efficacy. Scores for perceived barriers were retained so that higher scores represented higher perceived barriers. This change was made because we hypothesised that higher perceived barriers would increase screening hesitancy, whilst higher perceived risk, benefits and efficacy scores would reduce hesitancy. IMD decile was categorised as low (deciles 1-5) or high (deciles 6-10). A P value $<.05$ (two-tailed) was considered statistically significant for all analyses. Statistical analyses were performed using SPSS 23.0 (IBM, Chicago, IL)

\section{Results}

165/281 questionnaires were returned (58\% response rate). The characteristics of participants and nonparticipants are shown in Table 1. Compared to non-participants, participants were more likely to be 
female $(p<0.01)$, older $(p<0.01)$ and living in a less deprived area $(<0.05)$.

Table 1

Characteristics of participants and non-participants

\begin{tabular}{|lllll|}
\hline & $\begin{array}{l}\text { Participants } \\
(\mathbf{n = 1 6 5 )}\end{array}$ & $\begin{array}{l}\text { Non-participants } \\
(\mathbf{1 1 6})\end{array}$ & $\boldsymbol{p}$ value & $\begin{array}{c}\text { Effect } \\
\text { size }\end{array}$ \\
\hline Gender & & & & \\
Male & $69(42 \%)$ & $68(59 \%)$ & $<0.01$ & 0.16 \\
Female & $96(58 \%)$ & $48(41 \%)$ & & \\
\hline Age (median) & 55 & 49 & $<0.01$ & 0.54 \\
\hline $\begin{array}{l}\text { Index of multiple deprivation (IMD) } \\
\text { decilea (median) }\end{array}$ & 7 & 5 & $<0.05$ & 0.28 \\
\hline $\begin{array}{l}\text { alMD deciles were calculated by postcode using the English IMD 2019 data (276 recipients), } \\
\text { Welsh 2019 data (4 recipients) and Scottish 2020 IMD data (1 recipient). }\end{array}$ & \\
\hline
\end{tabular}

\section{Participants at excess risk of lung cancer:}

159 out of 165 participants were considered to be at risk of lung cancer based on their treatment for $\mathrm{HL}$ (prior receipt of an alkylating agent known to increase lung cancer risk and/or a radiation dose to the lung.) Subsequent data presented in this paper refers to these 159 individuals. The median age was 55, $60 \%$ were female, $92 \%$ were of white British ethnicity, $38 \%$ were current or former smokers and $7 \%$ were current smokers. The median number of years since diagnosis and last HL treatment was 24 and 23 years respectively. In terms of treatment for $\mathrm{HL}, 144$ (90.5\%) had received radiotherapy, which was most commonly delivered to the mediastinum, and 150 (94\%) had received chemotherapy of whom $62 \%$ had received alkylating agents known to increase lung cancer risk (procarbazine or mechlorethamine (also known as mustine)). The cause of excess lung cancer risk was a combination of an alkylating agent and radiation to the lung in $49 \%$, radiation alone in $41.5 \%$ and alkylating agent alone in $9.5 \%$. The demographic and clinical features of the study participants at risk of lung cancer are shown in Table 2. 
Table 2

Characteristics of participants at excess risk of lung cancer

\section{Clinical and demographic features of participants at excess risk of lung cancer $n=159$}

Current age: median (range) $\quad 55$ (29-80)

Gender

Female: 96 (60.3\%)

Male: $64(39.7 \%)$

Ethnicity

White British: 147 (92\%)

Other ${ }^{\mathrm{a}}: 12(8 \%)$

Level of education $(n=156)$

Education below university level: 86 (54.7\%)

University educated: 57 (37\%)

No educational qualifications: $13(8.3 \%)$

Employment $(n=158)$

Full or part time employed (or in full time education / training): $101(64 \%)$

Retired: 40 (25.3\%)

Other: $17(10.7 \%)$

HL classification

Classical HL: 150 (94\%)

Nodular lymphocyte predominant HL: 9 (6\%)

Years since diagnosis: median $24(6-48)$

(range)

Time since

last treatment: median (range)

Sites of radiation (lung and non-lung) $n=144$

$23(6-44)$

Mediastinal +/- other area: 95 (66\%)

Mantle field +/- other area: 28 (19\%)

Other area: 21 (15\%)

Chemotherapy regimens ${ }^{b}$

ChIVPP/EVA only: 46 (31\%)

$(n=150)$

ABVD only: $43(29 \%)$

MVPP only: $19(13 \%)$

Multiple chemotherapy regimens: 32 (21\%) (of whom 17 underwent stem cell transplant and of whom 28 received procarbazine or mechlorethamine)

VAPEC-B only: $10(6 \%)$ 


\section{Clinical and demographic features of participants at excess risk of lung cancer $n=159$}

Cause of excess lung cancer risk by treatment modality
Radiation to lung and alkylating agent: 78 (49\%)

Radiation to lung only: 66 (41.5\%)

Alkylating agent only: 15 (9.5\%)

Never smokers: 96 (62\%)

Former smokers: 49 (31\%)

Current smokers: $12(7 \%)$

Family history of lung cancer $(n=159)$

In parents or siblings: $12(8 \%)$

Another family member: 20 (13\%)

Self-rated health $(n=157)$

Good/fair: 97 (61.8\%)

Poor/very poor: 14 (8.9\%)

Revised Life-Orientation Test scores (possible range 0-24): median (range)

$15(0-23)$

a Other ethnicities: 2 Indian, 2 Irish, 1 White and Black Caribbean, 1 Mixed (Arab and British), 2 Arab, 1 Bangladesh, 1 African, 1 Caribbean, 1 East African and Asian

${ }^{b}$ ChIVPP-EVA: chlorambucil, vinblastine, procarbazine, prednisolone, etoposide, vincristine, doxorubicin, ABVD: doxorubicin, bleomycin, vinblastine, prednisolone), MVPP: mechlorethamine, vinblastine, procarbazine, prednisolone, VAPEC-B: doxorubicin, cyclophosphamide, etoposide, vincristine, bleomycin, prednisolone

\section{Lung cancer knowledge, beliefs and willingness to be screened}

$31 \%$ of participants selected lung cancer as being a late effect of treatment from the list of health conditions. 82/158 (52\%) of participants who answered the question about comparative risk of lung cancer believed that their personal risk was higher than the average person of their age and sex, 43 (27.2\%) believed they were at equal risk, 8 (5\%) at lower risk and 25 (16\%) did not know. 52/158 (33\%) of participants (32 women, 20 men) had previously been invited to undergo bowel cancer screening, of whom $40(77 \%)$ had taken up the offer at least once (25 women, 15 men). Among female participants, $90 / 95$ (95\%) had previously been invited to undergo breast cancer screening, of whom 89 (99\%) had taken up the offer at least once. Possible score ranges, median scores, range and interquartile range for the perceived risk, perceived benefits, perceived barriers and self-efficacy scales are shown in Table 3 . 
Table 3

Lung cancer screening health belief scale scores

\begin{tabular}{|ll|}
\hline & Median (range; IQR) \\
\hline Perceived risk score (possible range 3-15) $n=159$ & $9(3-15 ; 3)$ \\
\hline Perceived benefits score (possible range 6-30) =158 & $24(11-30 ; 5)$ \\
\hline Perceived barriers score in ever smokers (possible range 15-75) $n=59$ & $23(15-61 ; 14)$ \\
\hline Perceived barriers score in never smokers (possible range 12-60) $n=94$ & $16(12-40 ; 10)$ \\
\hline Self-efficacy score (possible range 7-28) $n=157$ & $28(17-28 ; 3)$ \\
\hline
\end{tabular}

Out of 157 participants who answered the question 'If you were invited to go for a lung cancer screening test, would you go?' 127 (81\%) responded 'yes, definitely', 27 (17\%) responded 'yes, probably' and 3 (2\%) responded 'probably not'. There were no distinct commonalities among the three participants who indicated that they would probably not attend lung cancer screening compared to those responding yes probably/definitely. The single female responder who would probably not attend lung cancer screening was among the $1 \%$ of participants who had not participated in breast cancer screening despite being invited.

PLCOall2014 scores were calculable for 130 participants. The median 6-year lung cancer risk was $0.09 \%$ $(<0.001-8.2 \%)$. Thirteen (10\%) participants - who were all former or current smokers - met the risk threshold for screening ( $\geq 1.51 \%$ ), but when the age bracket for lung cancer screening in the UK (55-74) was applied, just $6 \%$ would be eligible for lung cancer screening in the UK through pilots aimed at ever smokers.

A logistic regression analysis was performed to identify factors associated with lung cancer screening hesitancy. 158 participants with complete data for the dependant and independent variables were included in the model. The overall model was statistically significant when compared to the null model $(p<0.01)$, explained $59 \%$ of the variation in screening willingness and correctly predicted $90.5 \%$ of cases. On univariate analysis, the following factors were associated with screening hesitancy: being male (odds ratio (OR) 2.52, confidence interval $(\mathrm{Cl})$ 1.13-5.61) $\mathrm{p}<0.05$ ), lower perceived benefits (OR 1.29 $\mathrm{Cl} 1.14-1.47$ $\mathrm{P}<0.01)$, higher perceived barriers $(\mathrm{OR} 1.09 \mathrm{Cl} 1.05-1.15 \mathrm{p}<0.01)$ and lower self-efficacy $(\mathrm{OR} 1.45, \mathrm{Cl} 1.27-$ $1.65, p<0.01)$.

On multivariable analysis, the following factors were associated with screening hesitancy: being male (OR 5.94 Cl 1.64-21.44, $p<0.01$ ), living in an area with a high IMD decile (deciles 6-10) (OR 8.22 Cl 1.59$42.58, p<0.05)$ and lower levels of self-efficacy (OR $1.64 \mathrm{Cl} 1.30-2.08 \mathrm{p}<0.01)$. The results of the univariable and multivariable analyses are shown in Table 4. 
Table 4

Factors associated with lung cancer screening hesitancy $(n=158)$

\begin{tabular}{|lllllll|}
\hline & \multicolumn{3}{c}{ Univariable } & \multicolumn{3}{l|}{ Multivariable } \\
\hline Variable & $\begin{array}{l}\text { Odds } \\
\text { ratio }\end{array}$ & $\begin{array}{l}\text { Confidence } \\
\text { interval }\end{array}$ & $\begin{array}{l}\text { p } \\
\text { value }\end{array}$ & $\begin{array}{l}\text { Odds } \\
\text { ratio }\end{array}$ & $\begin{array}{c}\text { Confidence } \\
\text { interval }\end{array}$ & $\begin{array}{c}\text { p } \\
\text { value }\end{array}$ \\
\hline Male gender & $\mathbf{2 . 5 2}$ & $\mathbf{1 . 1 3 - 5 . 6 1}$ & $<0.05$ & $\mathbf{5 . 9 4}$ & $\mathbf{1 . 6 4 - 2 1 . 4 4}$ & $<0.01$ \\
\hline Age & 1.01 & $0.95-1.05$ & 0.41 & 0.99 & $0.92-1.06$ & 0.87 \\
\hline Years since treatment & 0.98 & $0.93-1.03$ & 0.44 & 0.91 & $0.83-1.00$ & 0.06 \\
\hline LOT-R score & 1.04 & $0.95-1.14$ & 0.31 & 1.18 & $0.97-1.43$ & 0.08 \\
\hline $\begin{array}{l}\text { Living in an area with a high } \\
\text { IMD decile }\end{array}$ & 1.43 & $0.61-3.37$ & 0.40 & $\mathbf{8 . 2 2}$ & $\mathbf{1 . 5 9 - 4 2 . 5 8}$ & $<0.05$ \\
\hline $\begin{array}{l}\text { No family history of lung } \\
\text { cancer }\end{array}$ & 1.23 & $0.25-5.96$ & 0.78 & 0.17 & $0.01-2.20$ & 0.17 \\
\hline Never smoker & 1.21 & $0.53-2.74$ & 0.64 & 0.80 & $0.20-3.17$ & 0.76 \\
\hline Cancer worry severity score & 0.84 & $0.59-1.20$ & 0.35 & 1.01 & $0.51-1.98$ & 0.97 \\
\hline Self-rated health score & 1.11 & $0.75-1.63$ & 0.59 & 0.60 & $0.27-1.31$ & 0.20 \\
\hline Lower perceived risk & 1.17 & $\mathbf{0 . 9 8 - 1 . 4 0}$ & 0.08 & 1.03 & $0.73-1.45$ & 0.82 \\
\hline Lower perceived benefits & $\mathbf{1 . 2 9}$ & $\mathbf{1 . 1 4 - 1 . 4 7}$ & $<0.01$ & 1.23 & $0.98-1.53$ & 0.06 \\
\hline Higher perceived barriers & $\mathbf{1 . 0 9}$ & $\mathbf{1 . 0 3 - 1 . 1 5}$ & $<0.01$ & 1.03 & $0.95-1.12$ & 0.37 \\
\hline Lower self-efficacy & $\mathbf{1 . 4 5}$ & $\mathbf{1 . 2 7 - 1 . 6 5}$ & $<0.01$ & $\mathbf{1 . 6 4}$ & $\mathbf{1 . 3 0 - 2 . 0 8}$ & $<\mathbf{0 . 0 1}$ \\
\hline
\end{tabular}

\section{Discussion}

In this questionnaire study, a large majority of long-term HL survivor respondents at risk of lung cancer indicated willingness to undergo lung cancer screening, were the test available. The motivations for lung cancer screening reported by participants in our previous qualitative study[18] - namely perceived benefits and desire for reassurance in a population exhibiting high levels of health anxiety - may explain the high levels of positive lung cancer screening intentions reported in this current study.

Upon registration in the ADAPT programme - usually 5 years following completion of treatment - our standard departmental policy provides a written treatment summary to all patients, including information about an excess risk of lung cancer to $\mathrm{HL}$ patients treated with thoracic radiotherapy. Although the vast majority of our participants would have received this information, only $31 \%$ recalled and selected lung cancer as being a potential late effect. A larger proportion (52\%) of our participants considered themselves to be at greater risk of lung cancer than the average person of the same age and sex. Knowledge of smoking as a lung cancer risk factor [35] and a perceptions by HL survivors of cancer 
treatments as being toxic [18] could have contributed to these comparative risk perceptions, particularly in participants who were not already aware that lung cancer can be a late effect of treatment. These findings demonstrate a lack of knowledge of personal lung cancer risk among HL survivors and reinforce the need for education about lung cancer risk upon invitation to a future lung cancer screening programme.

We hypothesised that few of our participants at risk of lung cancer would be eligible for screening through programmes aimed at ever smokers. Although the demographic characteristics of our participants do not fully reflect the HL survivor population overall (being older and female was associated with participation in the study), we found that just $10 \%$ met the $\geq 1.51 \%$-year lung cancer risk threshold for screening, falling to $6 \%$ when the age eligibility criteria for lung cancer screening in the UK were applied. This finding supports our hypothesis and provides justification for developing a lung cancer screening programme targeted towards $\mathrm{HL}$ survivors.

The demographic variables associated with screening hesitancy were being male and living in a less deprived area. The impact of gender may be explained by the very high levels of breast cancer screening uptake among female participants. Although cervical screening uptake was not investigated in this survey, it is likely that many of the female participants would also have experience of cervical screening. On the other hand, few male participants had been invited or participated in bowel cancer screening, potentially increasing hesitancy due to reduced levels of awareness around cancer screening and risk. Furthermore, our prior qualitative research found that women viewed breast cancer screening as a norm and their awareness of an excess risk of breast cancer aided their understanding of lung cancer risk, [18] perspectives which could have increased their willingness to undergo lung cancer screening. The association between living in a less deprived area and screening hesitancy in this study contrasts with the literature showing that a lower socioeconomic status is associated with lower cancer screening uptake.[19] This discrepancy may be due to this study investigating willingness to participate in a hypothetical screening scenario as opposed to actual lung cancer screening uptake. In reality, people living in more deprived areas are likely to experience greater barriers to participation than those in more affluent areas, such as the ability to take time off work and to travel to a screening appointment.

Overall, our participants exhibited high perceived benefits scores, high self-efficacy scores and low perceived barriers scores. We are not able to compare the scores of our participants with those of other groups firstly because we adapted the LCSHBS for our population and secondly because there is a lack of published studies that have used the scales in their intended population of ever smokers. In our study, the only health belief model construct predictive of screening hesitancy on multivariable analysis was selfefficacy. Self-efficacy is widely considered to be an important predictor of behaviour and is incorporated into numerous theoretical models. The question items relating to self-efficacy used in our study related to finding time to attend, transportation and ability to cope with anxiety about the results and uncertainty about the procedure. Our participants have prior experience of navigating the healthcare system experience which is likely to be ongoing for many due to the late effects of treatment - of undergoing 
scans and dealing with the associated anxiety. This prior experience and the fact that health is a priority for this group [12], [18] may explain the high levels of self-efficacy in our participants.

A meta-analysis of health belief model variables in predicting behaviour found that outcome expectancies - perceived benefits and barriers - were the strongest predictors of behaviour. [21] However, neither perceived benefits nor perceived barriers were associated with screening hesitancy in the multivariable analysis in our study. It is possible that outcome expectancies predict intention to decline lung cancer screening by HL survivors, but as there were very few participants who indicated they would decline screening, we could not perform this analysis. With regards to perceived risk, our findings are supported by the aforementioned meta-analysis which did not identify a correlation between susceptibility (perceived risk) and preventative behaviours.

\section{Strengths and limitations}

This study is the first to use quantitative methodology grounded in behavioural theory to explore the psycho-social factors predictive of willingness to undergo lung cancer screening among $\mathrm{HL}$ survivors. The study complements and supports our previous qualitative work on this topic and provides further evidence of high levels of willingness among this group to undergo lung cancer screening in the future. The resulting knowledge regarding the psycho-social factors which impact screening hesitancy could inform the design of a future lung cancer screening programme and its' associated informational materials, with the aim of optimising uptake rates. However, this must be balanced against the need to provide invitees with information about both the potential harms and benefits of screening in order to facilitate informed decision making.[36]

The extent to which the findings of this study can be applied to a national HL survivor population is limited by the characteristics of our participants who were registered in a long-term follow-up programme (most HL survivors who are in remission are discharged between 2-5 years after completion of treatment) and more likely to be female, older and living in a less deprived area than non-participants. In addition, a large majority of participants were of white British ethnicity and just over a third were university educated. Whilst the impact of gender and age on cancer screening participation rates is not always clear cut, a lower socioeconomic status (which correlates with lower levels of education and higher levels of smoking) has consistently been demonstrated to be a barrier to uptake [19] and people of non-white ethnicity face specific barriers to screening participation.[37] Therefore, the high levels of willingness to undergo lung cancer screening in this study may not reflect the entire HL survivor population, who would be expected to mirror the general population in terms of socioeconomic status and ethnicity. It is also possible that a greater proportion of non-participants to our study would decline lung cancer screening, compared to the participants. If this were the case, our study would have overestimated levels of willingness to undergo lung cancer screening. Although current smokers were poorly represented among our participants, the rates of current smoking in this study (7\%), mirror the findings of another study, [12] so it may be that rates of current smoking among $\mathrm{HL}$ survivors among our participants reflect those in the HL survivor population. 


\section{Conclusions And Directions For Future Research}

In this study we have identified the psycho-social factors associated with lung cancer screening hesitancy in HL survivors asked to consider a hypothetical lung cancer screening scenario and identified high levels of willingness to participate were lung cancer screening to become available. This study suggests that participation rates in lung cancer screening by $\mathrm{HL}$ survivors could be higher than in ever smokers and may exceed breast, cervical and bowel cancer screening uptake by the general population. Lung cancer screening is not routinely available for HL survivors and a trial of screening in this population is required to test lung cancer screening methodology established in ever smokers. Within such a trial, there would be value in exploring motivations and barriers to participation in a real-world setting. Further issues in this area worthy of exploration include developing lung cancer screening informational materials for $\mathrm{HL}$ survivors since current materials are aimed towards ever smokers and are not appropriate for use in this group. Developing a lung cancer risk calculator for this population is another important consideration to optimise selection criteria for lung cancer screening.

\section{Abbreviations}

HL: Hodgkin lymphoma

SMN : Subsequent malignant neoplasm

UK: United Kingdom

Cl: Cumulative incidence

CT: Computed tomography

NHS: National Health Service

HBM: Health Belief Model

LCSHBS: Lung cancer screening Health Belief Scales

PLCO: Prostate Lung Colorectal Ovarian

IMD: Index of Multiple Deprivation

OR: Odds ratio

Cl: Confidence Interval

\section{Declarations}

Ethics approval 
Ethical approval was given for this study by the Wales Research Ethics Committee 1 on $12^{\text {th }}$ March 2021 (reference 21/WA/0071).

\section{Consent to participate}

In accordance with the Health Research Authority's guidance on informed consent, return of the postal study questionnaire was taken to indicate informed consent.

\section{Consent for publication}

Not applicable

\section{Availability of data and materials}

All data generated or analysed during this study are included in this published article [and its supplementary information files].

\section{Competing Interests}

The authors declare that they have no competing interests.

\section{Funding}

Rachel Broadbent and Christopher J Armitage are supported by the National Institute for Health Research (NIHR) Manchester Biomedical Research Centre and the NIHR Greater Manchester Patient Safety Translational Research Centre (PSTRC-2016-003). Rachel Broadbent is also supported by the Christie Charity. The views expressed are those of the author(s) and not necessarily those of the NIHR or the Department of Health and Social Care.

\section{Author contributions}

RB led on study conception and design, supported by CJA, PC, JR and KL who all made substantial intellectual contributions towards the study protocol, questionnaire design, analysis plan and interpretation of the data. Material preparation, data collection and analysis were performed by RB. The first draft of the manuscript was written by RB. CJA, PC, JR, KL commented on the first draft and all authors (RB, CJA, PC, JR and $K L)$ reviewed and approved the final manuscript.

\section{Acknowledgements}

We would like to acknowledge data managers Emma Armstrong and Ellie White for their assistance with recruitment.

\section{References}


1. Cancer Research UK, "Hodgkin lymphoma statistics." [Online]. Available: https://www.cancerresearchuk.org/health-professional/cancer-statistics/statistics-by-cancertype/hodgkin-lymphoma\#heading-Zero. [Accessed: 20-Jun-2019].

2. Cancer Research UK, "Young people's cancers incidence statistics," 2019. [Online]. Available: https://www.cancerresearchuk.org/health-professional/cancer-statistics/young-peoplecancers/incidence. [Accessed: 03-Jul-2019].

3. L. B. Travis et al., "Lung cancer following chemotherapy and radiotherapy for Hodgkin's disease.," J. Natl. Cancer Inst., vol. 94, no. 3, pp. 182-192, 2002.

4. M. Schaapveld et al., "Cause-specific mortality among patients with Hodgkin lymphoma (HL) up to 40 years after treatment," J. Clin. Oncol., vol. 32, no. 15, 2014.

5. C. J. Bright et al., "Risk of subsequent primary neoplasms in survivors of adolescent and young adult cancer (Teenage and Young Adult Cancer Survivor Study): a population-based, cohort study.," Lancet. Oncol., vol. 20, no. 4, pp. 531-545, 2019.

6. B. M. P. Aleman, A. W. van den Belt-Dusebout, W. J. Klokman, M. B. van't Veer, H. Bartelink, and F. E. van Leeuwen, "Long-Term Cause-Specific Mortality of Patients Treated for Hodgkin's Disease," J. Clin. Oncol., vol. 21, no. 18, pp. 3431-3439, Sep. 2003.

7. A. K. Ng et al., "Long-term survival and competing causes of death in patients with early-stage Hodgkin's disease treated at age 50 or younger.," J. Clin. Oncol., vol. 20, no. 8, pp. 2101-8, Apr. 2002.

8. Public Health england, "Protocols for surveillance of women at very high risk of developing breast cancer," 2020. [Online]. Available: https://www.gov.uk/government/publications/breast-screeninghigher-risk-women-surveillance-protocols/protocols-for-surveillance-of-women-at-higher-risk-ofdeveloping-breast-cancer\#fn:1.

9. National Lung Screening Trial Research Team et al., "Reduced lung-cancer mortality with low-dose computed tomographic screening.," N. Engl. J. Med., vol. 365, no. 5, pp. 395-409, Aug. 2011.

10. H. J. De Koning et al., "Reduced Lung-Cancer Mortality with Volume CT Screening in a Randomized Trial," N. Engl. J. Med., vol. 386, no. 6, pp. 503-513, 2020.

11. NHS England - National Cancer Programme, "Targeted Screening for Lung Cancer with Low Radiation Dose Computed Tomography Standard Protocol prepared for the Targeted Lung Health Checks Programme," 2019. [Online]. Available: https://www.england.nhs.uk/publication/targetedscreening-for-lung-cancer/. [Accessed: 29-Jul-2019].

12. A. K. Ng et al., "Health Practice in Long-Term Survivors of Hodgkin's Lymphoma," Int. J. Radiat. Oncol. Biol. Phys., vol. 71, no. 2, pp. 468-476, 2008.

13. J. Waller, K. Osborne, and J. Wardle, "Enthusiasm for cancer screening in Great Britain: A general population survey," Br. J. Cancer, vol. 112, no. 3, pp. 562-566, 2015.

14. Nuffield Trust, “Cancer screening," 2021. [Online]. Available: https://www.nuffieldtrust.org.uk/resource/breast-and-cervical-cancer-screening. [Accessed: 11-Oct2021]. 
15. S. L. Quaife et al., "Lung Screen Uptake Trial (LSUT): Randomised Controlled Trial Testing Targeted Invitation Materials.," Am. J. Respir. Crit. Care Med., pp. 1-60, 2019.

16. M. Corkum, J. A. Hayden, G. Kephart, R. Urquhart, C. Schlievert, and G. Porter, "Screening for new primary cancers in cancer survivors compared to non-cancer controls: a systematic review and metaanalysis.," J. Cancer Surviv., vol. 7, no. 3, pp. 455-63, Sep. 2013.

17. A. Uhlig, J. Mei, I. Baik, C. Meyer, and J. Uhlig, "Screening utilization among cancer survivors: a metaanalysis," J. Public Health (Bangkok)., vol. 40, no. 1, pp. 129-137, Mar. 2018.

18. R. Broadbent, T. Seale, C. J. Armitage, J. Radford, and K. Linton, "The perspectives of survivors of Hodgkin lymphoma on lung cancer screening: A qualitative study," Heal. Expect., 2021.

19. E. A. Sarma, M. I. Silver, S. C. Kobrin, P. M. Marcus, and R. A. Ferrer, "Cancer screening: health impact, prevalence, correlates, and interventions," Psychol. Heal., vol. 34, no. 9, pp. 1036-1072, 2019.

20. C. Abraham and P. Sheeran, "The Health Belief Model," in Predicting Health Behaviour, 2nd ed., M. Conner and P. Norman, Eds. Open University Press, 2005, pp. 28-80.

21. C. J. Carpenter, "A meta-analysis of the effectiveness of health belief model variables in predicting behavior," Health Commun., vol. 25, no. 8, pp. 661-669, 2010.

22. A. Miles, S. Rainbow, and C. von Wagner, "Cancer Fatalism and Poor Self-Rated Health Mediate the Association between Socioeconomic Status and Uptake of Colorectal Cancer Screening in England," Cancer Epidemiol. Biomarkers Prev., vol. 20, no. 10, pp. 2132-2140, Oct. 2011.

23. J. Wardle et al., "Psychosocial influences on older adults' interest in participating in bowel cancer screening," Prev. Med. (Baltim)., vol. 31, no. 4, pp. 323-334, 2000.

24. C. Vrinten, J. Waller, C. Von Wagner, and J. Wardle, "Cancer Fear: Facilitator and Deterrent to Participation in Colorectal Cancer Screening," Cancer Epidemiol. Biomarkers Prev., vol. 24, no. 2, pp. 400-405, Feb. 2015.

25. J. Wardle, K. Robb, S. Vernon, and J. Waller, "Screening for prevention and early diagnosis of cancer," Am. Psychol., vol. 70, no. 2, pp. 119-133, Feb. 2015.

26. N. Ali et al., "Barriers to uptake among high-risk individuals declining participation in lung cancer screening: A mixed methods analysis of the UK lung cancer screening (UKLS) trial," BMJ Open, vol. 5, no. 7, 2015.

27. S. L. Quaife et al., "Smokers' interest in a lung cancer screening programme: A national survey in England," BMC Cancer, vol. 18, no. 1, pp. 1-10, 2018.

28. L. Carter-Harris, J. E. Slaven, P. Monohan, and S. M. Rawl, "Development and psychometric evaluation of the lung cancer screening health belief scales," Cancer Nursing, vol. 40, no. 3. pp. 237244, 01-May-2017.

29. R. Gramling, D. Anthony, G. Frierson, and D. Bowen, "The cancer worry chart: A single-item screening measure of worry about developing breast cancer," Psychooncology., vol. 16, no. 6, pp. 593-597, Jun. 2007. 
30. J. D. Jensen, J. K. Bernat, L. A. Davis, and R. Yale, "Dispositional cancer worry: Convergent, divergent, and predictive validity of existing scales," J. Psychosoc. Oncol., vol. 28, no. 5, pp. 470-489, Sep. 2010.

31. M. F. Scheier, C. S. Carver, and M. W. Bridges, "Distinguishing optimism from neuroticism (and trait anxiety, self-mastery, and self-esteem): A reevaluation of the Life Orientation Test.," J. Pers. Soc. Psychol., vol. 67, no. 6, pp. 1063-1078, 1994.

32. J. E. WARE, M. KOSINSKI, and S. D. KELLER, "A 12-Item Short-Form Health Survey: Construction of Scales and Preliminary Tests of Reliability and Validity," Med. Care, vol. 34, no. 3, 1996.

33. R. Bränström, S. Kristjansson, and H. Ullén, "Risk perception, optimistic bias, and readiness to change sun related behaviour," Eur. J. Public Health, vol. 16, no. 5, pp. 492-497, Oct. 2006.

34. M. C. Tammemägi et al., "Evaluation of the Lung Cancer Risks at Which to Screen Ever- and NeverSmokers: Screening Rules Applied to the PLCO and NLST Cohorts," PLOS Med., vol. 11, no. 12, 2014.

35. E. R. Park et al., "A qualitative study of lung cancer risk perceptions and smoking beliefs among national lung screening trial participants," Nicotine Tob. Res., vol. 16, no. 2, pp. 166-173, 2014.

36. NHS Cancer Screening Programmes, "Consent to Cancer Screening," Sheffield, 2009.

37. B. Young, L. Bedford, D. Kendrick, K. Vedhara, J. F. R. Robertson, and R. Das Nair, "Factors influencing the decision to attend screening for cancer in the UK: A meta-ethnography of qualitative research," $J$. Public Heal. (United Kingdom), vol. 40, no. 2, pp. 315-339, 2018.

\section{Supplementary Files}

This is a list of supplementary files associated with this preprint. Click to download.

- Studyquestionnaire.docx 